\author{
D. Utebaev ${ }^{1, *}$, G.Kh. Utepbergenova ${ }^{1}$, K.O. Tleuov ${ }^{2}$ \\ ${ }^{1}$ Berdakh Karakalpak State University, Nukus, Uzbekistan; \\ ${ }^{2}$ Al-Khwarizmi Nukus branch of the Tashkent University of Information Technologies, Nukus, Uzbekistan \\ (E-mail:dutebaev_56@mail.ru, utepbergenovagu@gmail.com,quwat80@mail.ru)
}

\title{
On convergence of schemes of finite element method of high accuracy for the equation of heat and moisture transfer
}

\begin{abstract}
In this paper difference schemes of the finite element method of a high order of accuracy for the nonstationary equation of moisture transfer of Aller are constructed and investigated. The increased order of accuracy is achieved through special sampling of temporal and spatial variables. The stability and convergence of the constructed numerical algorithms are proved, the corresponding a priori estimates are obtained in various norms, which are used later to obtain estimates of the accuracy of the scheme under weak assumptions on the smoothness of solutions to the differential problem.
\end{abstract}

Keywords: Aller's equation, finite element method, difference schemes, stability, a priori estimates, convergence, accuracy.

\section{Introduction}

As it is known, research in the field of heat and moisture transfer is fundamental in solving many applied problems, for example, problems of hydrogeology, agrophysics, ecology, building physics, etc. [1]. The interaction of heat fluxes in the soil-ground and snow cover determines the processes of infiltration, migration and frost heaving, evaporation and transpiration, metamorphism and snow melting. These processes determine conditions for overwintering and growing crops. In addition, the role of moisture migration and infiltration in the formation of productive moisture reserves in agricultural fields is of great importance. Mathematical models of these processes are described mainly by the Aller or Aller-Lykov moisture transfer equation [2]. This paper considers numerical methods for solving boundary value problems for the Aller moisture transfer equation written in a more general form. In this case, difference schemes of the finite element method of the fourth order of accuracy, constructed and investigated in [3], are used. These schemes have certain advantages over other schemes: a) high order accuracy scheme (above two); b) in addition to the solution itself, its derivative (velocity) is simultaneously found with the same accuracy; c) using interpolation representation

$$
\begin{gathered}
y(t)=y^{n} \phi_{00}^{n}(t)+\dot{y}^{n} \phi_{10}^{n}(t)+y^{n+1} \phi_{01}^{n}(t)+\dot{y}^{n+1} \phi_{11}^{n}(t), \\
\phi_{00}^{n}(t)=2 \xi^{3}-3 \xi^{2}+1, \quad \phi_{01}^{n}(t)=3 \xi^{2}-2 \xi^{3}, \phi_{10}^{n}(t)=\tau\left(\xi^{3}-2 \xi^{2}+\xi\right), \phi_{11}^{n}(t)=\tau\left(\xi^{3}-\xi^{2}\right),
\end{gathered}
$$

if necessary, it is possible to get a solution and its derivative at any time; d) since the scheme is two-layer, it is possible to use a variable step without loss of accuracy; e) the scheme is conditionally stable and requires 4 times more arithmetic operations than the schemes of the finite difference method, but this scheme allows to choose large time steps to achieve a certain accuracy. To obtain an estimate of the accuracy a special technique for obtaining a priori estimates is used. The classical approach to the study of the convergence of difference schemes based on the Taylor formula imposes high requirements on the smoothness of the desired solution. Recently a number of results have been obtained on the estimation of the rate of convergence of difference schemes for equations of mathematical physics. These results can be found in [4-8]. Similar studies for various non-stationary problems were carried out by the authors in [3, 9-11].

\footnotetext{
${ }^{*}$ Corresponding author.

E-mail: dutebaev_56@mail.ru
} 


\section{Statement of the problem}

The problems of the thermal and water regime of the root layer of the soil, evaporation, transpiration, etc. are described by the following equation (the Aller equation in general form) [1]

$$
\frac{\partial u}{\partial t}=L_{1} u+\sigma \frac{\partial}{\partial t}\left(L_{2} u\right)+f(x, t), \quad(x, t) \in Q_{T}=\{x \in \Omega, 0<t \leq T\} .
$$

Here $\Omega=\left\{x \mid x=\left(x_{1}, x_{2}\right), 0<x_{\alpha}<l_{\alpha}, \alpha=1,2\right\}$,

$$
\begin{gathered}
L_{m} u=\sum_{\alpha=1}^{p_{m}} \frac{\partial}{\partial x_{\alpha}}\left(k_{\alpha}^{m}(x) \frac{\partial u}{\partial x_{\alpha}}\right)-q^{m}(x), \quad x \in \Omega, p_{m}=1,2, \ldots, \\
0<k_{0} \leq k_{\alpha}^{m}(x) \leq k_{1}, q^{m}(x) \geq 0, m=1,2,
\end{gathered}
$$

where $\sigma, k_{0}, k_{1}$ are positive constants.

For equation (2) the initial condition

$$
u(x, 0)=u_{0}(x), \quad x \in \Omega
$$

is set and some local or non-local boundary conditions are given.

Local conditions are classical boundary conditions, for example, the first boundary value condition

$$
u(x, t)=0, \quad x \in \partial \Omega, \quad t \in(0, T] .
$$

Conditions are called non-local if the boundary conditions are relations connecting the values of the sought solution and its derivatives at the boundary and interior points of the domain. Similar conditions arise in the mathematical modelling of processes of various natural phenomenon, for example, in the study of problems of moisture transfer, thermal conductivity, mathematical biology, control, etc. For example, for equation (1) in the one-dimensional case, the non-local boundary conditions

$$
u(0, t)=\lambda u(l, t), \quad u_{x}(0, t)=\lambda u_{x}(l, t), \quad t \in[0, T]
$$

are given in $[1-2]$.

Let us formulate a generalized statement of problem (2)-(4). We call the generalized solution of problem (2)-(4) as the function $u(x, t)$, in which each $t \in[0, T]$ belongs to Sobolev space $H=\stackrel{\circ}{W_{2}^{1}}(\Omega)$, has a derivative $\frac{\partial u}{\partial t} \in L_{2}(0, T)$ and satisfies the relations [12]

$$
\left(\frac{d u(t)}{d t}, \vartheta\right)+\sigma a_{1}\left(\frac{d u(t)}{d t}, \vartheta\right)+a_{2}(u(t), \vartheta)=(f(t), \vartheta), \forall \vartheta(x) \in H, u(0)=u_{0}
$$

almost everywhere on $(0, T)$. Here

$$
a_{m}(u(t), \vartheta)=-\left(L_{m} u, \vartheta\right)=\int_{\Omega} \sum_{\alpha=1}^{p_{m}}\left(k^{m}(x) \frac{\partial u}{\partial x_{\alpha}} \cdot \frac{\partial \vartheta}{\partial x_{\alpha}}+q^{m}(x) u \vartheta\right) d x, \quad m=1,2 .
$$

For bilinear form $a_{m}(u, \vartheta)$ there is an evaluation $a_{m}(\vartheta, \vartheta) \geq k\|\vartheta\|^{2}$.

Note that the dimension of the operators $L_{1}, L_{2}$ can be different, i.e., $p_{1} \neq p_{2}$, and so $L_{1}$ can be strongly elliptical and $L_{2}$ can be a degenerate operator that does not contain all second derivatives of variables $x_{\alpha}$.

\section{Discretization in space and time}

We discretize problem (2)-(3) with respect to spatial variables using the finite element method. Let $H_{h} \subset H$ be many elements of the form $\vartheta_{h}=\sum_{i=1}^{N} a_{i} \phi_{i}(x)$. Here $\left\{\phi_{i}=\phi_{i}(x)\right\}_{i=1}^{N}$ is the basis of piecewise polynomial functions that are polynomials of degree on each finite element (a segment in one-dimensional case, a triangle or rectangle in two-dimensional case, etc.). 
Let us write relation (5) a semi-discrete problem for $t \in[0, T]$ :

$$
\begin{gathered}
\left(\frac{d u_{h}(t)}{d t}, \vartheta_{h}\right)+\sigma a_{1}\left(\frac{d u_{h}}{d t}, \vartheta_{h}\right)+a_{2}\left(u_{h}(t), \vartheta_{h}\right)=\left(f(t), \vartheta_{h}\right), \quad \forall \vartheta_{h} \in H_{h}, \\
u_{h}(0)=u_{0, h} .
\end{gathered}
$$

Problem (6) corresponds to the Cauchy problem in time for the system of ordinary differential equations of the first order for coefficients of the approximate solution $u_{h}(t)=\sum_{i=1}^{N} a_{i}(t) \phi_{i}$ from $H_{h}$ :

$$
M \frac{d \vec{a}(t)}{d t}+G \vec{a}(t)=\vec{\Phi}(t), \quad \vec{a}(0)=\vec{a}^{0} .
$$

Here, $\vec{a}(t)=\left\{a_{i}(t)\right\}_{i=1}^{N}, \quad\left\{\vec{a}_{i}(0)\right\}_{i=1}^{N}$ are dimension vectors $N ; M=\left\{\left(\phi_{i}, \phi_{j}\right)\right\}_{i, j=1}^{N}$ is the mass matrix, $G=\left\{a\left(\phi_{i}, \phi_{j}\right)\right\}_{i, j=1}^{N}$ is the stiffness matrix and $\vec{\Phi}(t)=\{\Phi(t)\}_{i=1}^{N}$ is a vector of the right side.

The same problem can also be written in the form of an operator equation

$$
\begin{gathered}
D \frac{d u_{h}(t)}{d t}+A u_{h}(t)=f_{h}(t), u_{h}(0)=u_{0, h}, \\
u_{h}(0)=u_{0, h} .
\end{gathered}
$$

Here $u_{h}(t)$ is the element of finite-dimensional space $H_{h}$ for any moment in time $t$, operators $D$ and $A$ operate from $H_{h}$ to $H_{h}: D=M+\sigma G_{1}, A=G_{2}, M=\left(\left(\phi_{i}, \phi_{j}\right)\right)_{i, j=1}^{N}$ is a subspace coordinate system mass matrix $H_{h}$, and $G_{m}=\left(a_{m}\left(\phi_{i}, \phi_{j}\right)\right)_{i, j=1}^{N}$ is a stiffness matrix corresponding to the operator $L_{m} u, m=1,2$ in $H_{h}$.

We approximate problem (7) with a three-parameter finite element method of the fourth order of accuracy in time [3]:

$$
\left\{\begin{array}{l}
D \frac{\hat{y}-y}{\tau}-\frac{\tau^{2}}{12} A \frac{\hat{y}-\dot{y}}{\tau}+A \frac{\hat{y}+y}{2}=\phi_{1}, \\
\gamma D \frac{\hat{y}-\dot{y}}{\tau}+\alpha A \frac{\hat{y}-y}{\tau}+\beta A \frac{\hat{y}+\dot{y}}{2}=\phi_{2} \\
y^{0}=u_{0}, \dot{y}^{0}=u_{1}
\end{array}\right.
$$

where

$$
\begin{gathered}
y=y^{n}=y\left(t_{n}\right), \hat{y}=y^{n+1}=y\left(t_{n}+\tau\right), \dot{y}=\dot{y}^{n}=\frac{d y}{d t}\left(t_{n}\right), \hat{\dot{y}}=\dot{y}^{n+1}=\frac{d y}{d t}\left(t_{n}+\tau\right), \\
\phi_{1}=\frac{1}{\tau} \int_{t_{n}}^{t_{n+1}} f(t) d t, \quad \phi_{2}=\frac{12}{\tau^{3}} \int_{t_{n}}^{t_{n+1}} f(t)\left(s_{1} \vartheta_{2}^{(1)}+s_{2} \vartheta_{2}^{(3)}\right) d t, \\
s_{1}=15 \gamma-35 \alpha / 3, \quad s_{2}=140 \gamma-350 \alpha / 3, \\
v_{2}^{(1)}=\tau(\xi+1 / 2), \quad v_{2}^{(3)}=\tau\left(\xi^{3}-3 \xi^{2} / 2+\xi / 2\right), \quad \xi=\left(t-t_{n}\right) / \tau .
\end{gathered}
$$

Scheme (8) obeys the condition of the fourth order of approximation in time

$$
\alpha+\beta=\gamma, \quad \alpha>0, \quad 0<\beta \leq \alpha /(3 \varepsilon), \quad \alpha, \beta=O\left(\tau^{2}\right), 0<\varepsilon<1 .
$$

Circuit stability conditions are

$$
\alpha=\tau^{2} / 12, \quad \beta>0, \quad \gamma>0, \quad, \quad \bar{R} \geq((1+\varepsilon) / 4) \bar{A},
$$

where $\bar{R}=\frac{1}{\tau}\left(\gamma D^{2}+\frac{\tau^{2}}{12}(3 \beta+\alpha) A^{2}\right), \quad \bar{A}=\tau \beta A^{2}$.

A high order of accuracy of the scheme is achieved due to a special discretization of temporal and spatial variables [3]. 


\section{Investigation of the accuracy of discretization in space}

Let us estimate the accuracy of the solution of problem (2)-(4). All notations are borrowed from [13]. The following theorem holds.

Theorem 1 Let $u(x, t) \in L_{2}\left\{[0, T] ; \quad W_{2}^{k+1}(\Omega) \cap \stackrel{\circ}{W} \underset{2}{1}(\Omega)\right\}$. If a narrowing of space $H_{h}$ into a single finite element is a polynomial of degree $k$, then for the solution of problem (7) there is an estimation of accuracy

$$
\begin{aligned}
& \sqrt{\int_{0}^{t}\left\|u\left(x, t^{\prime}\right)-u_{h}\left(x, t^{\prime}\right)\right\|_{0}^{2} d t^{\prime}}+\sigma \sqrt{\int_{0}^{t}\left\|u\left(x, t^{\prime}\right)-u_{h}\left(x, t^{\prime}\right)\right\|_{1}^{2} d t^{\prime}} \\
& +\left\|\int_{0}^{t}\left[u\left(x, t^{\prime}\right)-u_{h}\left(x, t^{\prime}\right)\right] d t^{\prime}\right\|_{1}^{t} \leq M h^{k}\left\{\|u(x, 0)\|_{k}+\sigma\|u(x, 0)\|_{k+1}\right. \\
& \left.+\sqrt{(1+\sigma) \int_{0}^{t}\left\|u\left(x, t^{\prime}\right)\right\|_{k+1}^{2} d t^{\prime}}\right\}, \quad \forall t \in[0, T], M=M\left(k_{0}, k_{1}, T\right) .
\end{aligned}
$$

Proof. We integrate identity (5) over $t$ from $t_{n}=n \tau, n=0,1, \ldots$ to $t_{n+1}=t_{n}+\tau$ and apply the formula for integration by parts

$$
\begin{gathered}
\int_{t_{n}}^{t_{n+1}}\left[-(u(t), \dot{\vartheta})-\sigma a_{1}(u(t), \dot{\vartheta})+a_{2}(u(t), \vartheta)\right] d t+\left.\left[(u(t), \vartheta)+\sigma a_{1}(u(t), \vartheta)\right]\right|_{t_{n}} ^{t_{n+1}} \\
=\int_{t_{n}}^{t_{n+1}}(f(t), \vartheta) d t, \forall \vartheta(x) \in H .
\end{gathered}
$$

Similar actions for identity (6) give

$$
\int_{t_{n}}^{t_{n+1}}\left[-\left(u_{h}, \dot{\vartheta}_{h}\right)-\sigma a_{1}\left(u_{h}, \dot{\vartheta}_{h}\right)+a_{2}\left(u_{h}, \vartheta_{h}\right)\right] d t+\left.\left[\left(u_{h}, \vartheta_{h}\right)+\sigma a_{1}\left(u_{h}, \vartheta_{h}\right)\right]\right|_{t_{n}} ^{t_{n+1}}=\int_{t_{n}}^{t_{n+1}}\left(f, \vartheta_{h}\right) d t, \forall \vartheta_{h}(x) \in H_{h} .
$$

Here and further $\dot{u}=\partial u / \partial t$. Choosing $\vartheta=\vartheta_{h} \in H_{h} \subset H$ in (11) and subtracting both obtained identities, we have

$$
\begin{aligned}
& \int_{t_{n}}^{t_{n+1}}\left[-\left(u-u_{h}, \dot{\vartheta}_{h}\right)-\sigma a_{1}\left(u-u_{h}, \dot{\vartheta}_{h}\right)+a_{2}\left(u-u_{h}, \vartheta_{h}\right)\right] d t \\
& +\left.\left[\left(u-u_{h}, \vartheta_{h}\right)+\sigma a_{1}\left(u-u_{h}, \vartheta_{h}\right)\right]\right|_{t_{n}} ^{t_{n+1}}=0, \quad \forall \vartheta_{h}(x) \in H_{h} .
\end{aligned}
$$

Let $z_{h}=u-u_{h}=e_{h}+\xi_{h}$. Let us choose a trial function

$$
\vartheta_{h}(t)=-\int_{t}^{s} \xi_{h}\left(t^{\prime}\right) d t^{\prime} \in H_{h}, t<s ; \quad \vartheta_{h}(t)=0, t \geq s, \dot{\vartheta}_{h}(t)=\xi_{h}(t), \vartheta_{h}(s)=0 .
$$

Taking into account the introduced designations, identity (12) can be written in the form:

$$
\begin{aligned}
& \int_{t_{n}}^{t_{n+1}}\left[-\left(\xi_{h}, \xi_{h}\right)-\sigma a_{1}\left(\xi_{h}, \xi_{h}\right)+a_{2}\left(\dot{\vartheta}_{h}, \vartheta_{h}\right)\right] d t+\left.\left[\left(\xi_{h}, \vartheta_{h}\right)+\sigma a_{1}\left(\xi_{h}, \vartheta_{h}\right)\right]\right|_{t_{n}} ^{t_{n+1}} \\
& =\int_{t_{n}}^{t_{n+1}}\left[\left(e_{h}, \xi_{h}\right)+\sigma a_{1}\left(e_{h}, \xi_{h}\right)-a_{2}\left(e_{h}, \vartheta_{h}\right)\right] d t-\left.\left[\left(e_{h}, \vartheta_{h}\right)+\sigma a_{1}\left(e_{h}, \vartheta_{h}\right)\right]\right|_{t_{n}} ^{t_{n+1}} .
\end{aligned}
$$


Since $a_{2}\left(\dot{\vartheta}_{h}, \vartheta_{h}\right)=\frac{1}{2} \frac{d}{d t} a_{2}\left(\vartheta_{h}, \vartheta_{h}\right)$, then the last identity can be written as:

$$
\begin{gathered}
-\int_{t_{n}}^{t_{n+1}}\left(\xi_{h}, \xi_{h}\right) d t-\sigma \int_{t_{n}}^{t_{n+1}} a_{1}\left(\xi_{h}, \xi_{h}\right) d t+\frac{1}{2} a_{2}\left(\vartheta_{h}, \vartheta_{h}\right)\left(t_{n+1}\right)+\left.\left[\left(\xi_{h}, \vartheta_{h}\right)+\sigma a_{1}\left(\xi_{h}, \vartheta_{h}\right)\right]\right|_{t_{n}} ^{t_{n+1}} \\
=-\left.\left[\left(e_{h}, \vartheta_{h}\right)+\sigma a_{1}\left(e_{h}, \vartheta_{h}\right)\right]\right|_{t_{n}} ^{t_{n+1}}+\frac{1}{2} a_{2}\left(\vartheta_{h}, \vartheta_{h}\right)\left(t_{n}\right)+\int_{t_{n}}^{t_{n+1}}\left[\left(e_{h}, \xi_{h}\right)+\sigma a_{1}\left(e_{h}, \xi_{h}\right)-a_{2}\left(e_{h}, \vartheta_{h}\right)\right] d t .
\end{gathered}
$$

Let us sum up (14) by $n=\overline{1, m-1}$, where is the number $m$ corresponds to the moment in time $s=m \tau$ :

$$
\begin{gathered}
-\int_{0}^{s}\left(\xi_{h}, \xi_{h}\right) d t-\sigma \int_{0}^{s} a_{1}\left(\xi_{h}, \xi_{h}\right) d t+\frac{1}{2} a_{2}\left(\vartheta_{h}, \vartheta_{h}\right)(s)+\left.\left[\left(\xi_{h}, \vartheta_{h}\right)+\sigma a_{1}\left(\xi_{h}, \vartheta_{h}\right)\right]\right|_{0} ^{s} \\
-\left.\left[\left(e_{h}, \vartheta_{h}\right)+\sigma a_{1}\left(h, \vartheta_{h}\right)\right]\right|_{0} ^{s}+\frac{1}{2} a_{2}\left(\vartheta_{h}, \vartheta_{h}\right)(0)+\int_{0}^{s}\left[\left(e_{h}, \xi_{h}\right)+\sigma a_{1}\left(e_{h}, \xi_{h}\right)-a_{2}\left(e_{h}, \vartheta_{h}\right)\right] d t .
\end{gathered}
$$

Considering the properties of the function $\vartheta_{h}(t)$ (see eq. (13)) and the initial condition $\xi_{h}(0)=0$, from the last identity we have

$$
\begin{gathered}
\int_{0}^{s}\left(\xi_{h}, \xi_{h}\right) d t+\sigma \int_{0}^{s} a_{1}\left(\xi_{h}, \xi_{h}\right) d t+\frac{1}{2} a_{2}\left(\vartheta_{h}, \vartheta_{h}\right)(0) \\
=-\left[\left(e_{h}, \vartheta_{h}\right)(0)+\sigma a_{1}\left(e_{h}, \vartheta_{h}\right)(0)\right]-\int_{0}^{s}\left[\left(e_{h}, \xi_{h}\right)+\sigma a_{1}\left(e_{h}, \xi_{h}\right)-a_{2}\left(e_{h}, \vartheta_{h}\right)\right] d t .
\end{gathered}
$$

Let us introduce one more function

$$
w_{h}(t)=\int_{0}^{t} \xi_{h}\left(t^{\prime}\right) d t^{\prime} \in H_{h}, t<s ; w_{h}(t)=0, t \geq s .
$$

Then $\vartheta_{h}(t)=w_{h}(t)-w_{h}(s)$, and, finally, we have the energy identity:

$$
\begin{gathered}
\int_{0}^{s}\left(\xi_{h}, \xi_{h}\right) d t+\sigma \int_{0}^{s} a_{1}\left(\xi_{h}, \xi_{h}\right) d t+\frac{1}{2} a_{2}\left(w_{h}, w_{h}\right)(s)=\left(e_{h}(0), w_{h}(s)\right) \\
+\sigma a_{1}\left(e_{h}(0), w_{h}(s)\right)-\int_{0}^{s}\left[\left(e_{h}, \xi_{h}\right)+\sigma a_{1}\left(e_{h}, \xi_{h}\right)-a_{2}\left(e_{h}, w_{h}(t)-w_{h}(s)\right)\right] d t .
\end{gathered}
$$

Let us estimate the terms on the right hand side of (15):

$$
\begin{aligned}
&\left(e_{h}(0), w_{h}(s)\right) \leq \varepsilon_{1}\left(w_{h}(s), w_{h}(s)\right)+\frac{1}{4 \varepsilon_{1}}\left(e_{h}(0), e_{h}(0)\right), \\
& a_{1}\left(e_{h}(0), w_{h}(s)\right) \leq \varepsilon_{2} a_{1}\left(w_{h}(s), w_{h}(s)\right)+\frac{1}{4 \varepsilon_{2}} a_{1}\left(e_{h}(0), e_{h}(0)\right), \\
&\left|\int_{0}^{s}\left(e_{h}, \xi_{h}\right) d t\right| \leq \varepsilon_{3} \int_{0}^{s}\left(\xi_{h}, \xi_{h}\right) d t+\frac{1}{4 \varepsilon_{3}} \int_{0}^{s}\left(e_{h}, e_{h}\right) d t, \\
&\left|\int_{0}^{s} a_{1}\left(e_{h}, \xi_{h}\right) d t\right| \leq \varepsilon_{4} \int_{0}^{s} a_{1}\left(\xi_{h}, \xi_{h}\right) d t+\frac{1}{4 \varepsilon_{4}} \int_{0}^{s} a_{1}\left(e_{h}, e_{h}\right) d t,
\end{aligned}
$$




$$
\begin{aligned}
& \left|\int_{0}^{s} a_{2}\left(e_{h}, w_{h}(t)-w_{h}(s)\right) d t\right| \\
& \leq \varepsilon_{5} \int_{0}^{s} a_{2}\left(w_{h}(t), w_{h}(t)\right) d t+s \varepsilon_{5} a_{2}\left(w_{h}(s), w_{h}(s)\right)+\frac{1}{2 \varepsilon_{5}} \int_{0}^{s} a_{2}\left(e_{h}, e_{h}\right) d t .
\end{aligned}
$$

Choosing $\varepsilon_{1}=\varepsilon_{2}=\varepsilon_{3}=\varepsilon_{4}=1 / 2$, and $\varepsilon_{5}$ from condition $\varepsilon_{1} / 2+\varepsilon_{5} T \leq 1 / 4$, from (15) we get the estimate

$$
\begin{gathered}
\int_{0}^{s}\left(\xi_{h}, \xi_{h}\right) d t+\sigma \int_{0}^{s} a_{1}\left(\xi_{h}, \xi_{h}\right) d t+a_{2}\left(w_{h}, w_{h}\right)(s) \\
\leq M\left(\int_{0}^{s} a_{2}\left(w_{h}, w_{h}\right)(t) d t+\left(e_{h}(0), e_{h}(0)\right)+\sigma a_{1}\left(e_{h}(0), e_{h}(0)\right)+\left(w_{h}(s), w_{h}(s)\right)\right. \\
\left.+\sigma a_{1}\left(w_{h}(s), w_{h}(s)\right)+\int_{0}^{s}\left(e_{h}, e_{h}\right) d t+\sigma \int_{0}^{s} a_{1}\left(e_{h}, e_{h}\right) d t+\int_{0}^{s} a_{2}\left(e_{h}, e_{h}\right) d t\right),
\end{gathered}
$$

where $M=\max (8,1 / T, 16 T)$. Applying Gronwall's lemma, we obtain the error estimate

$$
\begin{gathered}
\int_{0}^{s}\left(\xi_{h}, \xi_{h}\right) d t+\sigma \int_{0}^{s} a_{1}\left(\xi_{h}, \xi_{h}\right) d t+a_{2}\left(w_{h}, w_{h}\right)(s) \\
\leq M\left[\left(e_{h}(0), e_{h}(0)\right)+\sigma a_{1}\left(e_{h}(0), e_{h}(0)\right)\right]+\left(w_{h}(s), w_{h}(s)\right)+\sigma a_{1}\left(w_{h}(s), w_{h}(s)\right) \\
\left.+\int_{0}^{s}\left(e_{h}, e_{h}\right) d t+\sigma \int_{0}^{s} a_{1}\left(e_{h}, e_{h}\right) d t+\int_{0}^{s} a_{2}\left(e_{h}, e_{h}\right) d t\right) .
\end{gathered}
$$

It's obvious that $k_{0}\left\|w_{h}(s)\right\|_{1}^{2} \leq a_{m}\left(w_{h}, w_{h}\right)(s) \leq k_{1}\left\|w_{h}(s)\right\|_{1}^{2},\left(\xi_{h}, \xi_{h}\right)(s)=\left\|\xi_{h}(s)\right\|_{0}^{2}$. Therefore, we have the final estimate for the error

$$
\begin{aligned}
& \int_{0}^{s}\left\|\xi_{h}(s)\right\|_{0}^{2} d t+\sigma \int_{0}^{s}\left\|\xi_{h}(s)\right\|_{1}^{2} d t+\left\|\int_{0}^{s} \xi_{h}(t) d t\right\|_{1}^{2} \\
& \leq M\left(\left\|e_{h}(0)\right\|_{0}^{2}+\sigma\left\|e_{h}(0)\right\|_{1}^{2}+\int_{0}^{s}\left\|e_{h}(t)\right\|_{0}^{2} d t+\sigma \int_{0}^{s}\left\|e_{h}(t)\right\|_{1}^{2} d t+\int_{0}^{s}\left\|e_{h}(t)\right\|_{1}^{2} d t\right) .
\end{aligned}
$$

For solutions $u(x, t) \in W_{2}^{k+1}(\Omega), \forall t \in[0, T]$, there is an evaluation [13]:

$$
\begin{aligned}
& \left\|e_{h}(0)\right\|_{0} \leq M h^{k+1}\left\|u_{0}\right\|_{k},\left\|e_{h}(0)\right\|_{1} \leq M h^{k+1}\left\|u_{0}\right\|_{k+1}, \\
& \left\|e_{h}(t)\right\|_{0} \leq M h^{k+1}\|u(t)\|_{k},\left\|e_{h}(t)\right\|_{1} \leq M h^{k}\|u(t)\|_{k+1} .
\end{aligned}
$$

Therefore, based on (16) and the triangle inequality $\left\|z_{h}\right\| \leq\left\|e_{h}\right\|+\left\|\xi_{h}\right\|$ the statement of the theorem holds. The theorem uses the standart notation for the Sobolev space $W_{2}^{k+1}$ from [13].

\section{Accuracy research of discretization in time}

Let us now turn to the estimation of the discretization error for problem (7) in time. Investigation of the error in approximating scheme (8) using the Taylor formula, as already mentioned, leads to overestimated requirements for the smoothness of the solution to the original problem. An alternative to this method of estimating the accuracy is the application of the Bramble-Hilbert lemma. This method of accuracy estimation is the main one in the theory of the finite element method for solving elliptic equations [13-16]. We also note the paper [8], in which the Bramble-Hilbert lemma is used to estimate the accuracy of solving difference schemes for elliptic problems. 
Let us apply the Bramble-Hilbert lemma to estimate the accuracy of the solution to the original problem with respect to the time variable. Recall that the solution $u_{h}(t)$ of semi-discrete task $(7)$ for each $t$ is an element of the discrete subspace $u_{h}(t) \in H_{h}$.

The following theorem holds.

Theorem 2. Let $A^{*}=A>0, D^{*}=D>0, A D=D A$ and the conditions of approximation (9) and stability (10) are fulfilled. Then to solve the scheme (8) approximate solution to problem (7) such that $\frac{d^{4} u_{h}}{d t^{4}}(t) \in L_{2}[0, T]$ and the accuracy estimate

$$
\begin{gathered}
\sqrt{\int_{0}^{t}\left\|u_{h}\left(t^{\prime}\right)-y\left(t^{\prime}\right)\right\|_{0}^{2} d t^{\prime}}+\sigma \sqrt{\int_{0}^{t}\left\|u_{h}\left(t^{\prime}\right)-y\left(t^{\prime}\right)\right\|_{1}^{2} d t^{\prime}}+\left\|\int_{0}^{t}\left[u_{h}\left(t^{\prime}\right)-y\left(t^{\prime}\right)\right] d t^{\prime}\right\|_{1} \\
\leq M \tau^{4}\left\{\left\|u_{h}(0)\right\|_{0}+\sigma\left\|u_{h}(0)\right\|_{1}+\sqrt{(1+\sigma) \int_{0}^{t}\left\|\frac{d^{4} u_{h}}{d t^{4}}\left(t^{\prime}\right)\right\|_{1}^{2} d t^{\prime}}\right\}
\end{gathered}
$$

is correct.

Proof. Denote by $H_{\tau}$ argument function subspace $t$, which are a cubic Hermitian spline of the form (1) on the interval $\left[t_{n}, t_{n+1}\right], n=0,1,2, \ldots$. Consider scheme solution (8) to $y(t) \in H_{\tau}$. Simultaneously for each $t, y(t)$ is an element of the subspace $H_{h}$. Actually $y(x, t) \in H_{h}^{\tau}=H_{h} \otimes H_{\tau}$.

Difference scheme (8) corresponds to the following weak setting

$$
\begin{gathered}
\int_{t_{n}}^{t_{n+1}}\left[-\left(y(t), \dot{\vartheta}_{\tau}\right)-\sigma a_{1}\left(y(t), \dot{\vartheta}_{\tau}\right)+a_{2}\left(y(t), \vartheta_{\tau}\right)\right] d t \\
+\left.\left[\left(y(t), \vartheta_{\tau}\right)+\sigma a_{1}\left(y(t), \vartheta_{\tau}\right)\right]\right|_{t_{n}} ^{t_{n+1}}=\int_{t_{n}}^{t_{n+1}}\left(f, \vartheta_{\tau}\right) d t, \quad \forall \vartheta_{\tau}(x) \in H_{h}^{\tau},
\end{gathered}
$$

where $y(t)$ is the cubic Hermitian spline (1).

In (18) select

$$
\vartheta_{\tau}(t)=-\int_{t}^{s} \xi_{\tau}(t) d t^{\prime}, t<s ; \vartheta_{\tau}(t)=0, t \geq s .
$$

It's clear that $\dot{\vartheta}_{\tau}(t)=\xi_{\tau}(t), t<s$ and $\vartheta_{\tau}(s)=0$. Substituting the function $\vartheta_{\tau}(t)$ into (18) and performing same transformations with the resulting identity that we used when evaluating $z_{h}=u-u_{h}=e_{h}+\xi_{h}$, we get the following energy identity

$$
\begin{gathered}
\int_{0}^{s}\left(\xi_{\tau}, \xi_{\tau}\right) d t+\sigma \int_{0}^{s} a_{1}\left(\xi_{\tau}, \xi_{\tau}\right) d t+\frac{1}{2} a_{2}\left(\vartheta_{\tau}, \vartheta_{\tau}\right)(0) \\
=\left(e_{\tau}, \vartheta_{\tau}\right)(0)+\sigma a_{1}\left(e_{\tau}, \vartheta_{\tau}\right)(0)-\int_{0}^{s}\left[\left(e_{\tau}, \xi_{\tau}\right)+\sigma a_{1}\left(e_{\tau}, \xi_{\tau}\right)-a_{2}\left(e_{\tau}, \vartheta_{\tau}\right)\right] d t .
\end{gathered}
$$

Denote by

$$
w_{\tau}(t)=\int_{0}^{t} \xi_{\tau}\left(t^{\prime}\right) d t^{\prime} \in H_{h} t<s, w_{\tau}(t)=0 t \geq s
$$

and note that $e_{\tau}(0)=u_{h}(0)-u_{I}^{\tau}(0)=u_{0, h}-u_{0, h}=0$. Then the last identity becomes

$$
\int_{0}^{t}\left(\xi_{\tau}, \xi_{\tau}\right) d t+\sigma \int_{0}^{t} a_{1}\left(\xi_{\tau}, \xi_{\tau}\right) d t+\frac{1}{2} a_{2}\left(w_{\tau}, w_{\tau}\right)(s)
$$

Mathematics series. № 2(102)/2021 


$$
=-\int_{0}^{s}\left[\left(e_{\tau}, \xi_{\tau}\right)+\sigma a_{1}\left(e_{\tau}, \xi_{\tau}\right)-a_{2}\left(e_{\tau}, w_{\tau}(t)-w_{\tau}(s)\right)\right] d t .
$$

Applying the Cauchy-Bunyakovsky inequality, $\varepsilon$-inequality and Gronwall's lemma, as in the estimate $\xi_{h}(t)$, we obtain from (19) the following estimate

$$
\begin{gathered}
\int_{0}^{s}\left\|\xi_{\tau}(t)\right\|_{0}^{2} d t+\sigma \int_{0}^{s}\left\|\xi_{\tau}(t)\right\|_{1}^{2} d t+\left\|\int_{0}^{s} \xi_{\tau}(t) d t\right\|_{1}^{2} \\
\leq M\left(\int_{0}^{s}\left\|e_{\tau}(t)\right\|_{0}^{2} d t+\sigma \int_{0}^{s}\left\|e_{\tau}(t)\right\|_{1}^{2} d t+\int_{0}^{s}\left\|e_{\tau}(t)\right\|_{1}^{2} d t\right) .
\end{gathered}
$$

Now let us estimate the error of the scheme $(8) \zeta_{\tau}(t)=\xi_{\tau}(t)+e_{\tau}(t)$. By the triangle inequality and $(a+b)^{2} \leq 2\left(a^{2}+b^{2}\right)$ we have

$$
\begin{gathered}
\int_{0}^{s}\left\|\zeta_{\tau}(s)\right\|_{0}^{2} d t+\sigma \int_{0}^{s}\left\|\zeta_{\tau}(s)\right\|_{1}^{2} d t+\left\|\int_{0}^{s} \zeta_{\tau}(t) d t\right\|_{1}^{2} \leq 2\left(\int_{0}^{s}\left\|e_{\tau}(s)\right\|_{0}^{2} d t+\sigma \int_{0}^{s}\left\|e_{\tau}(s)\right\|_{1}^{2} d t\right. \\
\left.+\left\|\int_{0}^{s} e_{\tau}(t) d t\right\|_{1}^{2}+\int_{0}^{s}\left\|e_{\tau}(s)\right\|_{0}^{2} d t+\sigma \int_{0}^{s}\left\|e_{\tau}(s)\right\|_{1}^{2} d t+\left\|\int_{0}^{s} e_{\tau}(t) d t\right\|_{1}^{2}\right) .
\end{gathered}
$$

For the last term, we apply the Cauchy-Bunyakovsky inequality and get

$$
\left\|\int_{0}^{s} e_{\tau}\left(t^{\prime}\right) d t^{\prime}\right\|_{1}^{2} \leq\left\|\sqrt{\int_{0}^{s} 1 d t^{\prime}} \sqrt{\int_{0}^{s} e_{\tau}^{2}\left(t^{\prime}\right) d t^{\prime}}\right\|_{1}^{2} \leq s \int_{0}^{s}\left\|e_{\tau}\left(t^{\prime}\right)\right\|_{1}^{2} d t^{\prime} .
$$

From this and (20) we have the estimate

$$
\begin{gathered}
\int_{0}^{s}\left\|\zeta_{\tau}(s)\right\|_{0}^{2} d t+\sigma \int_{0}^{s}\left\|\zeta_{\tau}(s)\right\|_{1}^{2} d t+\left\|\int_{0}^{s} \zeta_{\tau}(t) d t\right\|_{1}^{2} \\
\leq M\left(\left\|e_{\tau}(s)\right\|_{0}^{2}+\sigma\left\|e_{\tau}(s)\right\|_{1}^{2}+\int_{0}^{s}\left\|e_{\tau}(t)\right\|_{0}^{2} d t+(\sigma+1) \int_{0}^{s}\left\|e_{\tau}(t)\right\|_{1}^{2} d t\right) .
\end{gathered}
$$

Consider the linear functional $e_{\tau}\left(u_{h}\right)=u_{h}-u_{I}^{\tau}$. We introduce the change of variable $t=t_{n}+\eta \tau$,

$0<\eta<1$. Then, we get

$$
\tilde{e}_{\tau}\left(\tilde{u}_{h}(\eta)\right)=e_{\tau}\left(u_{h}\right)=u_{h}\left(t_{n}+\eta \tau\right)-u_{I}^{\tau}\left(t_{n}+\eta \tau\right)=\tilde{u}_{h}(\eta)-\tilde{u}_{I}^{\tau}(\eta) .
$$

This functionality is limited for continuous functions $\tilde{u}_{h}(\eta) \in C[0,1]$. Moreover, it is limited for $\tilde{u}_{h}(\eta) \in W_{2}^{4}[0,1]$. So it is written as

$$
\left|\tilde{e}_{\tau}\left(\tilde{u}_{h}\right)\right|=\left|\tilde{u}_{h}(\eta)-\tilde{u}_{I}^{\tau}(\eta)\right| \leq M \sum_{m=0}^{4}\left(\int_{0}^{1}\left(\frac{d^{m} \tilde{u}_{h}}{d \eta^{m}}\right)^{2} d \eta\right)^{1 / 2} .
$$

This functional vanishes on polynomials up to the third degree inclusive in the variable $\eta$, i.e., on the segment $[0,1] \tilde{u}_{I}^{\tau}$ a third-degree polynomial that interpolates $\tilde{u}_{h}$. Based on the Bramble-Hilbert lemma, from the last estimate one can obtain

$$
\left|\tilde{e}_{\tau}\left(\tilde{u}_{h}\right)\right|=\left|\tilde{u}_{h}(\eta)-\tilde{u}_{I}^{\tau}(\eta)\right| \leq \bar{M}\left(\int_{0}^{1}\left(\frac{d^{4} \tilde{u}_{h}}{d \eta^{4}}\right)^{2} d \eta\right)^{1 / 2} .
$$


Returning to the previous variables we have the estimate

$$
\left|e_{\tau}\left(u_{h}(t)\right)\right|=\left|u_{h}(t)-u_{I}^{\tau}(t)\right| \leq \bar{M} \tau^{7 / 2}\left(\int_{t_{n}}^{t_{n+1}}\left(\frac{d^{4} u_{h}}{d t^{4}}\right)^{2} d t\right)^{1 / 2}, \forall t \in\left[t_{n}, t_{n+1}\right] .
$$

Then

$$
\begin{gathered}
\int_{0}^{s}\left\|e_{\tau}\left(t^{\prime}\right)\right\|_{1}^{2} d t^{\prime}=\sum_{n=0}^{m-1} \int_{t_{n}}^{t_{n+1}}\left\|e_{\tau}\left(t^{\prime}\right)\right\|_{1}^{2} d t^{\prime} \leq \sum_{n=0}^{m-1} \int_{t_{n}}^{t_{n+1}} \bar{M}^{2} \tau^{7} \int_{t_{n}}^{t_{n+1}}\left\|\frac{d^{4} u_{h}}{d t^{4}}(t)\right\|_{1}^{2} d t d t^{\prime} \\
=\sum_{n=0}^{m-1} \bar{M}^{2} \tau^{8} \int_{t_{n}}^{t_{n+1}}\left\|\frac{d^{4} u_{h}}{d t^{4}}(t)\right\|_{1}^{2} d t=\bar{M}^{2} \tau^{8} \int_{0}^{s}\left\|\frac{d^{4} u_{h}}{d t^{4}}(t)\right\|_{1}^{2} d t .
\end{gathered}
$$

Similarly, the estimate

$$
\int_{0}^{s}\left\|e_{\tau}(t)\right\|_{0}^{2} d t \leq \bar{M}^{2} \tau^{8} \int_{0}^{s}\left\|\frac{d^{4} u_{h}}{d t^{4}}(t)\right\|_{0}^{2} d t
$$

holds. If limitations $\left\|\frac{d^{4} u_{h}}{d t^{4}}(t)\right\|_{0}$ are required for each $t$, then we obtain

$$
\begin{gathered}
\left\|e_{\tau}(s)\right\|_{0}^{2} \leq \bar{M}^{2} \tau^{7}\left\|\int_{t_{m-1}}^{t_{m}}\left(\frac{d^{4} u_{h}}{d t^{4}}\right)^{2} d t\right\|_{0} \leq \bar{M}^{2} \tau^{8} \max _{t}\left\|\frac{d^{4} u_{h}}{d t^{4}}\right\|_{0}^{2}, \\
\left\|e_{\tau}(s)\right\|_{1}^{2} \leq \bar{M}^{2} \tau^{8} \max _{t}\left\|\frac{d^{4} u_{h}}{d t^{4}}\right\|_{1}^{2} .
\end{gathered}
$$

Further, based on these estimates we obtain the statement of the theorem from (21).

\section{On the convergence of the scheme}

In order to estimate the approximation error we need to go from $u_{h}$ to the solution $u$ in the right-hand sides of $z=u-y=\left(u-u_{h}\right)-\left(y-u_{h}\right)$.

For $k=0,1$ the following estimate holds [13]:

$$
\left\|u_{h}\right\|_{k}=\left\|u-u+u_{h}\right\|_{k} \leq\|u\|_{k}+\left\|u-u_{h}\right\|_{k} \leq\|u\|_{k}+M h\|u\|_{k+1} \leq \bar{M}\|u\|_{k+1} .
$$

Therefore, estimate (17) has the form

$$
\begin{gathered}
\sqrt{\int_{0}^{t}\left\|u_{h}\left(t^{\prime}\right)-y\left(t^{\prime}\right)\right\|_{0}^{2} d t}+\sigma \sqrt{\int_{0}^{t}\left\|u_{h}\left(t^{\prime}\right)-y\left(t^{\prime}\right)\right\|_{1}^{2} d t}+\left\|\int_{0}^{t}\left[u_{h}\left(t^{\prime}\right)-y\left(t^{\prime}\right)\right] d t^{\prime}\right\|_{1} \\
\leq M \tau^{4}\left\{\|u(0)\|_{0}+\sigma\|u(0)\|_{1}+\sqrt{(\sigma+1) \int_{0}^{t}\left\|\frac{d^{4} u}{d t^{4}}\left(t^{\prime}\right)\right\|_{2}^{2} d t^{\prime}}\right\} .
\end{gathered}
$$

Thus, we formulate an assertion about the convergence of the solution of the vector scheme (8) to the solution of the original problem (2)-(4).

Theorem 3. Let $A^{*}=A>0, D^{*}=D>0, A D=D A$ and the conditions of approximation (9) and stability (10) of scheme (8). Then for its solution, which approximates the solution to problem (2)-(4) such that

$$
u(x, t) \in L_{2}\left\{[0, T] ; \quad W_{2}^{k+1}(\Omega) \cap \stackrel{\circ}{W} \frac{1}{2}(\Omega)\right\}, \frac{\partial^{4} u}{\partial t^{4}}(x, t) \in L_{2}\left\{[0, T] ; W_{2}^{2}(\Omega)\right\},
$$


the accuracy estimate

$$
\begin{aligned}
& \sqrt{\int_{0}^{t}\left\|u\left(x, t^{\prime}\right)-y\left(x, t^{\prime}\right)\right\|_{0}^{2} d t^{\prime}}+\sigma \sqrt{\int_{0}^{t}\left\|u\left(x, t^{\prime}\right)-y\left(x, t^{\prime}\right)\right\|_{1}^{2} d t^{\prime}}+\left\|\int_{0}^{t}\left[u\left(x, t^{\prime}\right)-y\left(x, t^{\prime}\right)\right] d t^{\prime}\right\|_{1} \\
& \leq M\left\{\tau^{4}\left(\|u(x, 0)\|_{0}+\sigma\|u(x, 0)\|_{1}+\sqrt{(1+\sigma) \int_{0}^{t}\left\|\frac{\partial^{4} u}{\partial t^{4}}\left(x, t^{\prime}\right)\right\|_{2}^{2} d t^{\prime}}\right)\right. \\
& \left.+h^{k}\left(\|u(x, 0)\|_{k}+\sigma\|u(x, 0)\|_{k+1}+\sqrt{(1+\sigma) \int_{0}^{t}\left\|u\left(x, t^{\prime}\right)\right\|_{k+1}^{2} d t^{\prime}}\right)\right\}
\end{aligned}
$$

is correct.

\section{Algorithm for implementing the scheme}

We consider one of the possible algorithms for implementing the scheme (8). We rewrite it as

$$
m_{11} \hat{y}+m_{12} \hat{\dot{y}}=\varphi_{1}, \quad m_{21} \hat{y}+m_{22} \hat{\dot{y}}=\varphi_{2},
$$

where

$$
\begin{gathered}
\varphi_{1}=\tau \phi_{1}+\left(D+\frac{\tau}{2} A\right) y-\frac{\tau^{2}}{12} A \dot{y}, \quad \varphi_{2}=\tau \phi_{2}+\alpha A y+\left(\gamma D+\frac{\tau}{2} \beta A\right) \dot{y} \\
m_{11}=D+\frac{\tau}{2} A, \quad m_{12}=-\frac{\tau^{2}}{12} A, \quad m_{21}=\alpha A, \quad m_{22}=\gamma D+\frac{\tau}{2} \beta A .
\end{gathered}
$$

Integrals in $\phi_{1}$ and $\phi_{2}$, for example, are calculated by Simpson's formula. Assuming that the operators $A$ and $D$ commute and excluding $\hat{\dot{y}}$ from equation $(22)$, we obtain

$$
C \hat{y}=F .
$$

Here $C=\gamma D^{2}+\frac{\tau}{2}(\beta+\gamma) A D+\frac{\tau^{2}}{12}(3 \beta+\alpha) A^{2}, \quad F=m_{22} \varphi_{1}-m_{12} \varphi_{2}$.

Equation (23) can be solved either directly by inverting the operator $C$ or by factoring it as

$$
C=\gamma C_{1} C_{2}=\gamma\left[D^{2}-\left(x_{1}+x_{2}\right) \tau A D+x_{1} x_{2} \tau^{2} A^{2}\right], \quad C_{k}=\left(D-x_{k} \tau A\right), \quad k=1,2
$$

Then equation (23) is solved using an algorithm

$$
\gamma_{1} C_{1} \bar{y}=F, \quad C_{2} \hat{y}=\bar{y}
$$

After finding $\hat{y}$ from (24) solution $\hat{\dot{y}}$ is calculated, for example, from the equation $\left(\gamma D+\frac{\tau}{2} \beta A\right) \hat{\dot{y}}=\varphi_{2}-\alpha A \hat{y}$.

\section{Conclusion}

Problems for the Aller moisture transfer equation are considered. On the basis of the finite element method difference schemes of high order of accuracy are constructed and investigated. The high order of accuracy of the circuit is achieved through special discretization of temporal and spatial variables. The convergence of the constructed algorithms is proved. Estimates for the accuracy of the scheme are obtained under weak assumptions on the smoothness of solutions to differential problems. Other boundary value problems can be studied similarly, in particular, nonlocal boundary value problems for equation (1). In addition, these results can be carried over to loaded equations with nonlocal boundary conditions.

\section{Remark}

A separate article will be devoted to computational experiments for test problems with local and non-local boundary conditions. 


\section{References}

1 Лыков А.В. Теория тепло- и массопереноса / А.В. Лыков, Ю.А. Михайлов. - М.-Л.: Гос. энерг. изд-во, 1963. - 535 с.

2 Нахушев А.М. Нагруженные уравнения и их применение / А.М. Нахушев. - М.: Наука, 2012. 232 c.

3 Москальков М.Н. Численное моделирование нестационарных процессов механики сплошной среды / М.Н. Москальков, Д. Утебаев. - Ташкент: Фан ва технология, 2012. - 160 с.

4 Djuraev I.N. On the Accuracy of the Method of Lines for Second-Order Quasilinear Hyperbolic Equations with a Small Parameter Multiplying the Highest Time Derivative / I.N. Djuraev, T.V. Kolesnik, V.L. Makarov // Differential Equations. - 1985. - Vol. 21. - No. 7. - P. 1164-1170.

5 Djuraev I.N. Investigation of the Convergence of the Solution of a Difference Scheme with Weights to the Generalized Solution of the Vibrating String Equation in the Class $W_{2}^{2}\left(Q_{T}\right) /$ I.N. Djuraev, M.N. Moskalkov // Differential Equations. - 1985. - Vol. 21. - No. 12. - P. 2145-2152.

6 Lazarov R.D. Difference Scheme of Second Order of Accuracy for the Axisymmetric Poisson Equation in Generalized Solutions / R.D. Lazarov, V.L. Makarov // Computational Mathematics and Mathematical Physics. - 1981. - Vol. 21. - No 5. - P. 95-107.

7 Москальков М.Н. Скорость сходимости методов дискретизации для волнового уравнения с обобщенными решениями / М.Н. Москальков, В.Л. Бурковская, И.Н. Джураев // Вычислительная и прикладная математика. - 1985. - № 57. - С. 26-33.

8 Самарский А.А. Разностные схемы для дифференциальных уравнений с обобщенными решениями / А.А. Самарский, Р.Д. Лазаров, В.Л. Макаров. - М.: Высш. шк., 1987. - 296 с.

9 Moskal'kov M.N. Convergence of the Finite Element Scheme for the Equation of Internal Waves / M.N. Moskal'kov, D. Utebaev // Cybernetics and Systems Analysis. - 2011. - Vol. 47. - No. 3. P. 459-465.

10 Moskalkov M.N. Convergence of Centered Difference Schemes for a System of Two-Dimensional Equations of Acoustics / M.N. Moskalkov, D. Utebaev // Journal of Mathematical Sciences. - 1992. - Vol. 58. No. 3. - P. 229-235.

11 Moskalkov M.N. Comparison of Some Methods for Solving the Internal Wave Propagation Problem in a Weakly Stratified Fluid / M.N. Moskalkov, D. Utebaev // Mathematical models and Computer Simulations. - 2011. - Vol. 3. - No.2 . - P. 264-271.

12 Марчук Г.И. Введение в проекционно-сеточные методы / Г.И. Марчук, В.И. Агошков. - М.: Наука, 1981. - $416 \mathrm{c}$.

13 Quarteroni A. Numerical Approximation of Partial Differential Equations / A. Quarteroni, A. Valli. Springer-Verlag, Berlin, 1994. - 544 p.

14 Strang G. An Analysis of the Finite Element Method / G. Strang, G. Fix. - Prentice-Hall, Inc., New Jersey, 1973.

15 CiarletP. The finite element method for elliptic problems / P. Ciarlet. - North-Holland Publishing Company, New York, 1978.

16 Mitchell A.R. The Finite Element Method in Partial Differential Equations / A.R. Mitchell, R. Wait. John Wiley and Sons, New York, 1977. 


\author{
Д. Отебаев ${ }^{1}$, Г.Х. Утепбергенова ${ }^{1}$, К.О. Тлеуов ${ }^{2}$ \\ ${ }^{1}$ Бердах атындавы Қарақалпақ мемлекеттік университеті, Нүкіс, Өзбекстан; \\ 2 Әл-Хорезми атындавы Ташкент ақпараттық, технологиялар \\ университетінін, Нүкістегі бөлімшесі, Нүкіс, Өзбекстан
}

\title{
Жылу-ылғал тасымалдау теңдеуі үшін жоғары дәлдікті ақырлы элементтер әдісінің схемасының жинақтылығы туралы
}

\begin{abstract}
Мақалада Аллердің ылғал тасымалдау бейстационарлық теңдеуі үшін жоғары дәлдіктегі ақырлы элементтер әдісінің айырымдық схемалары құрылып зерттелді. Дәлдіктің жоғарғы ретіне уақыт және кеңістіктік айнымалыларын арнайы дискретизациялау арқылы қол жеткізіледі. Құрылған сандық алгоритмдердің тұрақтылығы мен жинақтылығы дәлелденді, дифференциалдық есептің шешімдерінің тегістігі туралы әлсіз болжамдармен схеманың дәлдік бағалауларын алу үшін пайдаланылған, әртүрлі нормаларда сәйкес априорлық бағалаулар алынды.
\end{abstract}

Kiлm сөздер: Аллер теңдеуі, ақырлы элементтер әдісі, айырымдық схемалары, тұрақтылық, априорлық бағалаулар, жинақтылық, дәлдік.

\author{
Д. Утебаев ${ }^{1}$, Г.Х. Утепбергенова ${ }^{1}$, К.О. Тлеуов ${ }^{2}$ \\ ${ }^{1}$ Каракалпакский государственный университет им. Бердаха, Нукус, Узбекистан; \\ ${ }^{2}$ Нукусский филиал Ташкентского университета \\ информационных технологий им. алъ-Хорезми, Нукус, Узбекистан
}

\section{О сходимости схемы метода конечных элементов повышенной точности для уравнения тепло-влагопереноса}

В статье построены и исследованы разностные схемы метода конечных элементов высокого порядка точности для нестационарного уравнения влагопереноса Аллера. Повышенный порядок точности достигается за счет специальной дискретизации временных и пространственных переменных. Доказана устойчивость и сходимость построенных численных алгоритмов, получены соответствующие априорные оценки в различных нормах, которые использованы в дальнейшем для получения оценок точности схемы при слабых предположениях о гладкости решений дифференциальной задачи.

Ключевые слова: уравнение Аллера, метод конечных элементов, разностные схемы, устойчивость, априорные оценки, сходимость, точность.

\section{References}

1 Lykov, A.V., \& Mikhailov, A.V. (1963). Teoriia teplo- $i$ massoperenosa /Theory of heat and mass transfer]. Moscow - Leningrad: Gosudarstvennoe energeticheskoe izdatelstvo [in Russian].

2 Nakhushev, A.M. (2012). Nagruzhennye uravneniia $i$ ikh primenenie [Loaded equations and their application]. Moscow: Nauka [in Russian].

3 Moskalkov, M.N., \& Utebaev, D. (2012). Chislennoe modelirovanie nestatsionarnykh protsessov mekhaniki sploshnoi sredy [Numerical modeling of nonstationary processes in continuum mechanics]. Tashkent: Fan va tekhnologiia [in Russian].

4 Djuraev, I.N., Kolesni, T.V., \& Makarov, V.L. (1985). On the accuracy of the method of lines for secondorder quasilinear hyperbolic equations with a small parameter multiplying the highest time derivative. Differential Equations, 21(7), 1164-1170.

5 Djuraev, I.N., \& Moskalkov, M.N. (1985). Investigation of the convergence of the solution of a difference scheme with weights to the generalized solution of the vibrating string equation in the class $W_{2}^{2}\left(Q_{T}\right)$. Differential Equations, 21 (12), 2145-2152. 
6 Lazarov, R.D., \& Makarov, V.L. (1981). Difference scheme of second order of accuracy for the axisymmetric poisson equation in generalized solutions. Computational Mathematics and Mathematical Physics, $21(5), 95-107$.

7 Moskalkov, M.N., Burkovskaya, V.L., \& Djuraev, I.N. (1985). Skorost skhodimosti metodov diskretizatsii dlia volnovogo uravneniia s obobshchennymi resheniiami [Convergence rate of discretization methods for a wave equation with generalized solutions]. Vychislitelnaia i prikladnaia matematika - Computational and Applied Mathematics, 57, 26-33 [in Russian].

8 Samarskii, A.A., Lazarov, R.D., \& Makarov, V.L. (1987). Raznostnye skhemy dlia differentsialnykh uravnenii s obobshchennymi resheniiami [Difference schemes for differential equations with generalized solutions]. Moscow: Vysshaia shkola [in Russian].

9 Moskalkov, M.N., \& Utebaev, D. (2011). Convergence of the finite element scheme for the equation of internal waves. Cybernetics and Systems Analysis, 47(3), 459-465.

10 Moskalkov, M.N., \& Utebaev, D. (1992). Convergence of centered difference schemes for a system of two-dimensional equations of acoustics. Journal of Mathematical Sciences, 58(3), 229-235.

11 Moskalkov, M.N., \& Utebaev, D. (2011). Comparison of some methods for solving the internal wave propagation problem in a weakly stratified fluid .Mathematical models and Computer Simulations, 3(2), $264-271$.

12 Marchuk, G.I., \& Agoshkov, V.I. (1981). Vvedenie v proektsionno-setochnye metody [Introduction to projection-grid methods]. Moscow: Nauka [in Russian].

13 Quarteroni, A., \& Valli, A. (1994). Numerical Approximation of Partial Differential Equations. SpringerVerlag, Berlin.

14 Strang, G., \& Fix, G. (1973). An Analysis of the Finite Element Method. Prentice-Hall, Inc., New Jersey.

15 Ciarlet, P. (1978). The Finite Element Method for Elliptic Problems. North-Holland Publishing Company, New York.

16 Mitchell, A.R., \& Wait, R. (1977). The Finite Element Method in Partial Differential Equations. John Wiley and Sons, New York. 